\title{
Ingestión accidental de prótesis dental fija (cantilever) en paciente con historia de carcinoma de colon ${ }^{1}$
}

\section{Accidental swallowing of fixed denture (cantilever) in a patient with history of colon cancer}

\author{
Fonseca GM*
}

\section{RESUMEN}

Se presenta un caso de ingestión accidental de un puente de tipo cantilever metalocerámico de tres unidades en un paciente masculino de 51 años con historia de remoción quirúrgica de carcinoma colorrectal y colostomía provisoria (ano contranatura) para recuperación intestinal cuatro años antes. Aun cuando la naturaleza del objeto ingerido y las condiciones patológicas previas intestinales ponderan una conducta quirúrgica de remoción preventiva, dado el carácter asintomático y el control radiográfico del desplazamiento normal del objeto durante las primeras 24 horas, se optó por una conducta expectante que se resolvió finalmente con la excreción espontánea del mismo. Si bien la rehabilitación protésica fija debería brindar seguridad y estabilidad, un diseño sesgado del puente (indicación errónea del cantilever y pernos cortos) puede originar importantes daños potenciales (aspiración, perforación de esófago, absceso y fístula enterocólica, obstrucción intestinal), terapéuticas fuertemente invasivas e incluso la misma muerte del paciente, todos sucesos con claras connotaciones legales.

Palabras clave: Accidente, ingestión, prótesis fija, iatrogenia.

\section{SUMMARY}

This clinical report describes a case of accidental swallowing of three units cantilever porcelain fused metal bridge in a 51-years-old male with history of colon cancer and reversible colostomy four years ago. While the kind of the ingested foreign body and the prior gastrointestinal conditions recommend a surgical management decision to avoid the risks for perforation or obstruction, in our case the management decision was the conservative approach because of the asymptomatic condition and the radiographic observation of the normal pass the alimentary tract without complications. The fixed dentures should be secure and stable but an incorrect design of the bridge (wrong indication of cantilever; short post systems) are at increased risk of important potential harms (inhalation, oesophageal perforation, enterocolic fistula, intestinal obstruction), invasive intervention or even death, all with medicolegal consequences.

Key words: Accident, ingestion, fixed denture, iatrogenesis.

Fecha de recepción: 25 de abril de 2010.

Aceptado para publicación: 7 de mayo de 2010.

1 Trabajo subsidiado por Resolución Nº 69/08 de la Secretaría de Ciencia y Tecnología de la Universidad Nacional de Córdoba - S.E.C. y T.

* Doctor en Odontología. Profesor Titular Cátedra de Anatomía Patológica B. Director del Equipo de Pericias en Odontología Forense. Facultad de Odontología, Universidad Nacional de Córdoba, Argentina.

Fonseca FM. Ingestión accidental de prótesis dental fija (cantilever) en paciente con historia de carcinoma de colon. Av. Odontoestomatol 2011; 27 (6): 313-317. 


\section{INTRODUCCIÓN}

La ingestión accidental de cuerpos extraños es una situación de alta frecuencia en la consulta médica, tanto para la población pediátrica como para la adulta y pueden ser causa de morbilidad e incluso de mortalidad (1). En adultos mayores, se han reportado a las prótesis dentales, particularmente las parciales removibles, como los objetos más frecuentemente ingeridos en forma accidental $(1,2)$. Esto se ha considerado un factor determinante al momento de decidir la rehabilitación de dientes perdidos con prótesis fijas o removibles dada la mayor seguridad que las primeras presentan (2). Así mismo, y aún infrecuentemente reportada (3), la ingestión de prótesis fijas supone variadas causas entre las que se mencionan una retención inadecuada (4), fallos en el diseño de la misma (2) o específicos accidentes vinculados a intubación endotraqueal y anestesia general $(5,6)$.

La literatura ha referido que un $80 \%$ de los cuerpos extraños ingeridos logran atravesar sin dificultades el tracto gastrointestinal; sin embargo, un $20 \%$ va a necesitar de intervenciones no quirúrgicas para su remoción, o en el peor de los casos, un $1 \%$ van a requerir procedimientos invasivos para este fin (2, 7). En el caso de la ingestión de prótesis fijas múltiples (puentes), esto va a depender fundamentalmente de la conformación y el tamaño de dicho objeto (2). Se han puntualizado diferentes recomendaciones respecto de la conducta a seguir una vez reconocida la ingestión: en muchos casos la conducta expectante va a ser la ideal si hay ausencia de sintomatología (2), o incluso se ha enfatizado la remoción preventiva endoscópica del mismo ante una eventual obstrucción (6). Aun cuando se ha referido que los problemas mayores suceden a nivel de esófago y estómago y que una vez superado el píloro habitualmente no existen problemas en la excreción espontánea (2), en pacientes con cirugías previas gastrointestinales, malformaciones congénitas o patologías preexistentes, el riesgo de obstrucciones, perforaciones o hemorragias aumenta considerablemente, más aún si el objeto es metálico y con aristas, tal el caso de los puentes $(2,7,8)$. Es en esos casos que se ha enfatizado el retirar el objeto mediante endoscopias (6) o colonoscopias (9) para evitar una potencial obstrucción o daño intestinal. Se presenta un caso de ingestión accidental de un puente metalocerámico de 3 unidades en un paciente con historia de remoción quirúrgica de carcinoma de colon y la excreción espontánea del objeto. Se discuten además las probables implicaciones legales de dicho accidente.

\section{NOTA CLÍNICA}

Paciente masculino de 51 años de edad realiza la consulta telefónica de emergencia por ingestión accidental de un puente metalocerámico de tres unidades. Refiere que dicho puente posee una historia de 5 años en su colocación y que en dos oportunidades tuvo que ser recementado por desprendimiento espontáneo. En esta ocasión, el paciente menciona haber sentido movilidad de dicho puente por lo menos dos semanas anteriores al evento, pero por cuestiones personales no pudo asistir a la consulta con su profesional para el examen correspondiente y el recementado. Unas 6 horas después de acontecida la ingestión accidental, el paciente es asistido en servicio de guardia médica donde se le realizó una primera placa radiográfica abdominal. En ella se observa la presencia de un cuerpo radiopaco compatible con el objeto ingerido y ubicado en el estómago a nivel de su porción pilórica (Fig. 1, izquierda). Dada una historia reciente de remoción quirúrgica de carcinoma de colon y colostomía provisoria (ano contranatura) para recuperación intestinal cuatro años antes, el paciente evidencia un estado de importante ansiedad; sin embargo y ante la ausencia de síntomas, se le recomienda conducta expectante e ingestión de alimentos a base de fibra (acelga, espinaca) para facilitar la envoltura del bolo y el normal pasaje del cuerpo por el tracto. En control médico realizado a las 24 horas, el paciente refiere no tener molestias de ningún tipo y haber seguido cuidadosamente las instrucciones respecto a la dieta. Se realiza una segunda placa abdominal, la que muestra desplazamiento del objeto, ahora ubicado en la primera porción del intestino grueso (ciego) (Fig. 1, derecha). Esto mantiene la postura expectante, dada la progresión normal del objeto en el tracto gastrointestinal. Cuarenta y ocho horas después de sucedido el evento, se produce la expulsión natural del puente, el que es recuperado 


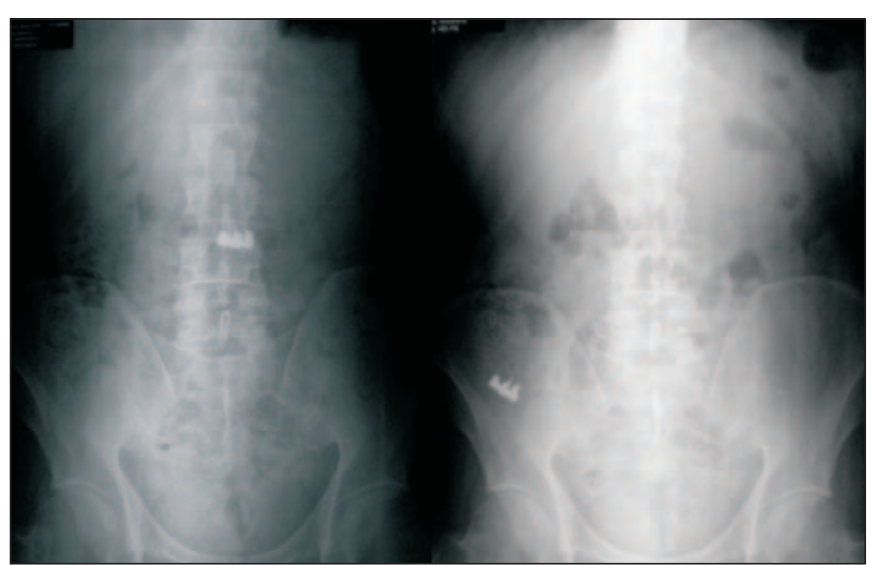

Fig. 1. Izquierda: radiografía abdominal 6 horas posteriores a la ingestión del objeto. Presencia de un cuerpo radiopaco compatible en forma y tamaño con el puente ingerido accidentalmente, ubicado en el estómago a nivel de su porción pilórica. Derecha: nueva radiografía abdominal 24 horas posteriores. Se observa desplazamiento del objeto, ahora ubicado en la primera porción del intestino grueso (ciego).

por el paciente y desinfectado en solución de hipoclorito de sodio durante 12 horas. Es en esta instancia que el paciente acude a esta consulta donde se realiza el examen extraoral del puente (Fig. 2): está compuesto por tres unidades con un diseño de tipo cantilever o "de extremo libre" con retención en primero y segundo premolares inferiores derechos y póntico en primer molar. Los pernos han permanecido cementados en el mismo puente, ambos con

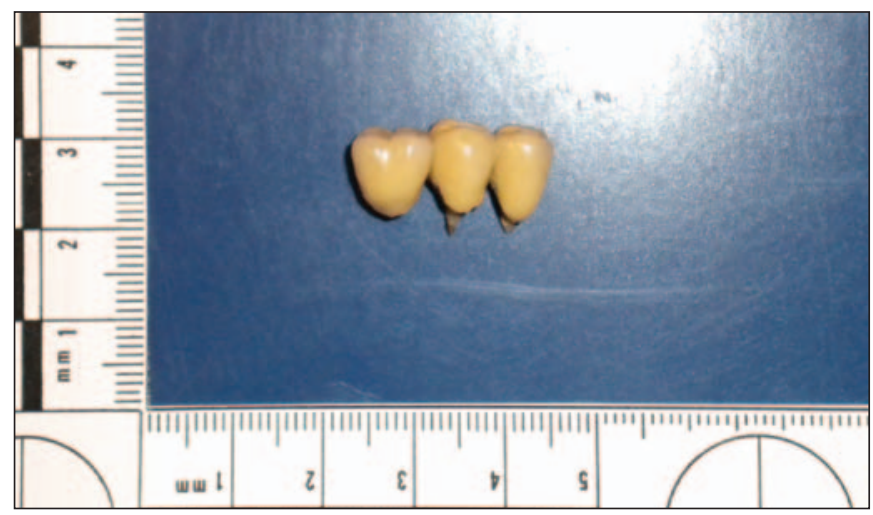

Fig. 2. Restauración protética fija recuperada post excreción espontánea 48 horas después de la ingestión accidental, compuesto por tres unidades con un diseño de tipo cantilever o "de extremo libre" (primer molar). Obsérvese que los pernos han permanecido cementados, ambos con una extensión inadecuada de entre 2 y $3 \mathrm{~mm}$. una extensión de entre 2 y $3 \mathrm{~mm}$. La exploración intraoral revela las raíces de ambos premolares en buen estado, con ausencia de filtraciones o caries. Ante la solicitud del paciente de recolocar el dispositivo, se realizan nuevos glaseado y recementado (Fig. 3), no sin antes especificarle que, dadas las condiciones irregulares de su confección, existe una alta probabilidad de que la situación se repita, a lo que el paciente da su conformidad explícita.

\section{DISCUSIÓN}

Neustein y Beicke (2007) (6) refieren que ante la ingestión accidental de una corona individual puede ser suficiente una conducta expectante y espera de excreción natural, pero si estos objetos poseen aristas metálicas o pónticos, aun asintomáticos, es recomendable la remoción mediante endoscopias o maniobras quirúrgicas dados los riesgos de obstrucción o daño intestinal, criterio compartido por algunos autores (7). Por otra parte, y coincidiendo con Eisen et al (2002) (8), Khouri y Dababneh (2004) (2), refieren que, una vez el objeto es descubierto, el clínico deberá sopesar el potencial daño según la ubicación del objeto y el riesgo de su remoción.

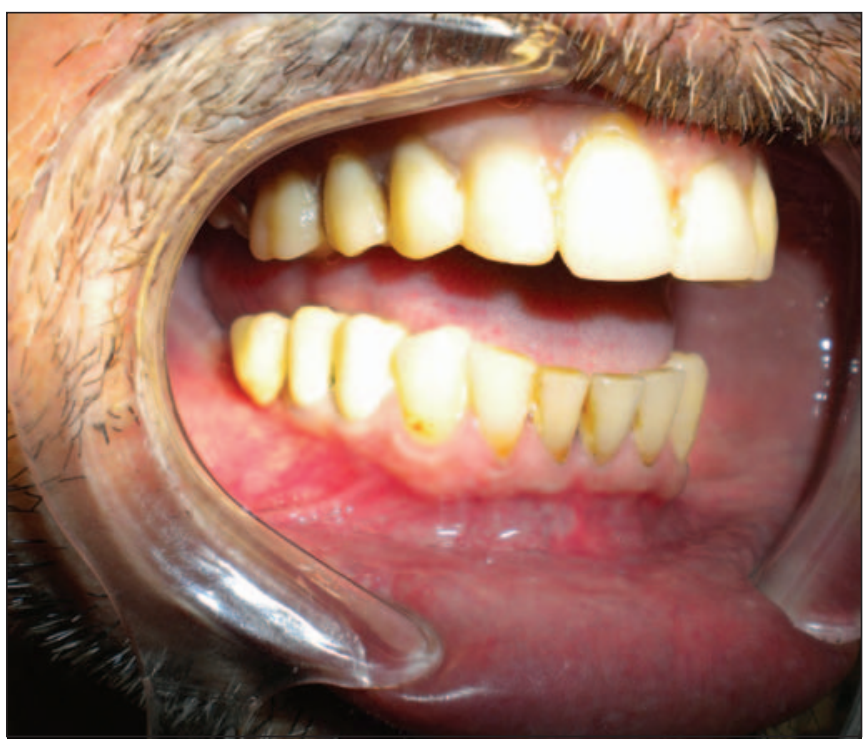

Fig. 3. Recementado del puente en boca ante la solicitud del paciente por razones estéticas. Dadas las condiciones irregulares de su confección, se solicitó su conformidad expresa explicitando los riesgos de un nuevo accidente. 
Estos autores, respecto de un caso de ingestión accidental de un puente de cuatro unidades donde la paciente se encontraba absolutamente asintomática (la radiografía abdominal fue conclusiva al no existir incluso certeza del accidente), concluyen que la ausencia de síntomas gástricos o intestinales puede definir una decisión de espera a la eventual expulsión. El puente fue excretado 48 horas después y recementado sin complicaciones de ninguna índole (2).

En ausencia de dientes pilares posteriores, la rehabilitación con pónticos cantilever se ha considerado una alternativa válida a los fines de evitar la implementación de prótesis removibles (10). Sin embargo, la literatura ha planteado sustanciales controversias respecto a la implementación de éstos, dado que tres factores primordiales deben ser cuidadosamente tenidos en cuenta: la selección de los pilares, el control de las fuerzas y la rigidez y extensión de los conectores (11). En el caso que se presenta, si bien las fuerzas aplicadas se encontraban por debajo de las normales (el paciente poseía prótesis completa superior removible acrílica), tanto la selección de los dientes pilares como los pernos en ellos, presentaban sesgos en sus diseños: ancho y largo del póntico eran mayores que los dientes pilares (11) y los pernos no presentaban los largos mínimos para ejercer una retención adecuada (12).

Si bien el caso expuesto tuvo una resolución feliz a una conducta negligente por parte del paciente (hacía dos semanas que el puente se había desprendido y se encontraba móvil e inestable en boca), lo real es que las implícitas seguridades de una rehabilitación fija no estaban cumpliéndose, y los daños potenciales (aspiración (13), perforación de esófago (14), absceso y fístula enterocólica (9), obstrucción intestinal), acrecentados en un paciente con historia patológica previa intestinal, podrían haber derivado en importantes lesiones, terapéuticas fuertemente invasivas e incluso la misma muerte del individuo. Phillips, citado por Hashmi y col (2004) (15) relata que en el Reino Unido, durante el periodo 1977-2003, unas 26 demandas fueron realizadas a odontólogos respecto de prótesis ingeridas accidentalmente. Si bien éstas fueron casi en su totalidad prótesis removibles, resulta necesario el mencionar que al momento de la publicación de ese trabajo, 13 de ellas ya tenían dictamen jurídico y de éstas, 6 (46,15\%) finalizaron favorables a los demandantes.

\section{BIBLIOGRAFÍA}

1. Haidary A, Leider JS, Silbergleit R. Unsuspected swallowing of a partial denture. AJNR Am J Neuroradiol 2007;28(9):1734-5.

2. A. Khouri, R. Dababneh. Accidental swallowing of a four unit anterior bridge: a case report. Jordanian Royal Medical Services JRMS 2004; 11(2):44-6.

3. Auluck A, Desai R. Accidental swallowing of a prosthesis. Dent Update 2008; 35 (8):577-9.

4. Beaumont RH. Retrieval of a swallowed casting 6 weeks after ingestion. A case report. Oral Surg Oral Med Oral Pathol 1987;64(3):287-8.

5. Lau G, Kulkarni V, Roberts GK, Brock-Utne J. "Where are my teeth?" A case of unnoticed ingestion of a dislodged fixed partial denture. Anesth Analg 2009;109(3):836-8.

6. Neustein S, Beicke M. Ingestion of a fixed partial denture during general anesthesia. Anesth Prog 2007;54(2):50-1.

7. Henderson CT, Engel J, Schlesinger P. Foreign body ingestion: Review and suggested guidelines for management. Endoscopy 1987;19(2):68-71.

8. Eisen GM, Baron TH, Dominitz JA, Faigel DO, Goldstein JL, Johanson JF et al; American Society for Gastrointestinal Endoscopy. Guideline for the management of ingested foreign bodies. Gastrointest Endosc 2002;55(7):802-6.

9. Tsai CY, Hsu CC, Chuah SK, Chiu KW, Changchien CS. Endoscopic removal of a dental prosthesis in the hepatic flexure of the colon. Chang Gung Med J 2003;26(11):843-6.

10. Crothers AJ, Wassell RW, Jepson N, Thomason JM. The use of cantilever bridges. Dent Update 1995;22(5):190-8. 
11. Hill EE. Decision-making for treatment planning a cantilevered fixed partial denture. Compend Contin Educ Dent 2009;30(9):580-5; quiz 586, 606.

12. Stockton LW. Factors affecting retention of post system: A literature review. J Prosthet Dent 1999; 81(4):380-5.

13. Tiwana KK, Morton T, Tiwana PS. Aspiration and ingestion in dental practice: a 10-year institutional review. J Am Dent Assoc 2004;135(9):1287-91.

14. Owen Korn B. Perforación esofágica. Causas y manifestaciones clínicas. Gastr Latinoam 2005; 16(2):133-136.
15. Hashmi S, Walter J, Smith W, Latis S. Swallowed partial dentures. J R Soc Med 2004; 97(2):72-5.

\section{CORRESPONDENCIA}

Dr. Gabriel Mario Fonseca Cátedra de Anatomía Patológica B Facultad de Odontología

Av. Haya de la Torre, s/n

Ciudad Universitaria

Córdoba (5000) Argentina.

e-mail: gabriel_fonseca@argentina.com 